\title{
Comment on "Out-of-plane equilibrium points in the restricted three-body problem with oblateness (Research Note)"
}

\author{
Nan $\mathrm{Wu}^{1}$, Xuefeng Wang ${ }^{1}$, and Li-Yong Zhou ${ }^{1,2}$ \\ ${ }^{1}$ School of Astronomy and Space Science, Nanjing University, Nanjing 210046, PR China \\ e-mail: zhouly@nju.edu.cn \\ ${ }^{2}$ Key Laboratory of Modern Astronomy and Astrophysics in Ministry of Education, Nanjing University, Nanjing 210046, \\ PR China
}

Received 2 January 2018 / Accepted 26 January 2018

\begin{abstract}
Douskos \& Markellos (2006, A\&A, 446, 357) first reported the existence of the out-of-plane equilibrium points in restricted three-body problem with oblateness. This result deviates significantly from the intuitive physical point of view that there is no other force that can balance the combined gravitation in $Z$ direction. In fact, the out-of-plane equilibrium in that model is illusory and we prove here that such equilibrium points arise from the improper application of the potential function.
\end{abstract}

Key words. celestial mechanics - minor planets, asteroids: general - methods: analytical

\section{Introduction}

In recent years, several papers by various authors have been devoted to investigating the properties of the out-of-plane equilibrium points, which are located outside the orbital plane of the oblate primary bodies in the restricted three-body problem. This research is mainly based on Douskos \& Markellos (2006), which first reported the existence of out-of-plane equilibrium points caused by the $J_{2}$ term perturbation.

Intuitively, in the $Z$ direction perpendicular to the orbital plane $(O-X Y)$, only gravitational forces from both primaries produce an acceleration towards the $O-X Y$ plane and no other force can balance such acceleration. Therefore, the existence of such out-of-plane equilibrium points is doubtful. We notice that even Douskos and Markellos suspected that the existence of such points is only a mathematical illusion due to the truncation of the potential.

For the case of oblate ellipsoidal primaries, we analytically show in this report that such equilibrium points proposed by Douskos \& Markellos (2006) are located just inside the Brillouin sphere, where the potential function adopted therein is invalid. These equilibrium points do not exist in the physical point of view.

\section{Estimation of the equilibrium}

Just as in Douskos \& Markellos (2006), let us consider the circular restricted three-body problem, in which a massless body $P$ moves under the gravitational force exerted by an oblate ellipsoid $m_{1}$ and a point mass $m_{2}$. We choose the distance between these two primaries as the unit of length, the total mass $\left(m_{1}+m_{2}\right)$ as the unit of mass, and the unit of time is chosen so as to make the gravitational constant $G=1$. The motion of the massless body in the synodic frame (see Fig. 1) can be described as

$$
\left\{\begin{array}{l}
\ddot{x}-2 n \dot{y}=\frac{\partial W}{\partial x} \\
\ddot{y}+2 n \dot{x}=\frac{\partial W}{\partial y}, \\
\ddot{z}=\frac{\partial W}{\partial z}
\end{array}\right.
$$

where $W$ is the potential function given by

$$
\begin{aligned}
W & =\frac{1}{2} n^{2}\left(x^{2}+y^{2}\right)+\frac{\mu_{1}}{r_{1}}\left[1+\frac{A}{2 r_{1}^{2}}\left(1-\frac{3 z^{2}}{r_{1}^{2}}\right)\right]+\frac{\mu_{2}}{r_{2}}, \\
\mu_{1} & =m_{1} /\left(m_{1}+m_{2}\right), \mu_{2}=1-\mu_{1}, \\
r_{1}^{2} & =\left(x+\mu_{1}\right)^{2}+y^{2}+z^{2}, \quad r_{2}^{2}=\left(x-\mu_{2}\right)^{2}+y^{2}+z^{2} .
\end{aligned}
$$

The angular velocity $n$ depends on the oblateness of $m_{1}$ and in this case $n=\sqrt{1+3 A / 2}$. The dimensionless quantity $A$ indicates the oblateness of $m_{1}$ which is defined as

$A=\frac{R_{e}^{2}-R_{p}^{2}}{5 D^{2}}=\frac{r_{e}^{2}-r_{p}^{2}}{5}, \quad r_{e}=\frac{R_{e}}{D}, r_{p}=\frac{R_{p}}{D}$,

where $D$ is the distance between two primaries, while $R_{e}\left(r_{e}\right)$ and $R_{p}\left(r_{p}\right)$ are the real (normalized) equatorial and polar radii of the ellipsoid $m_{1}$, respectively. It is worth noting that the potential of $m_{1}$ in $W$ is the truncation up to the $J_{2}$ term of the spherical harmonics expansion of the potential function.

Since we are only interested in the out-of-plane equilibrium points, we focus on the $Z$ direction, where the equilibrium is attained when $\partial W / \partial z=0$, i.e.,

$$
\frac{\partial W}{\partial z}=-\frac{\mu_{2}}{r_{2}^{3}} z-\frac{\mu_{1}}{r_{1}^{3}}\left[1+\frac{3 A}{2 r_{1}^{2}}\left(3-\frac{5 z^{2}}{r_{1}^{2}}\right)\right] z=0 .
$$




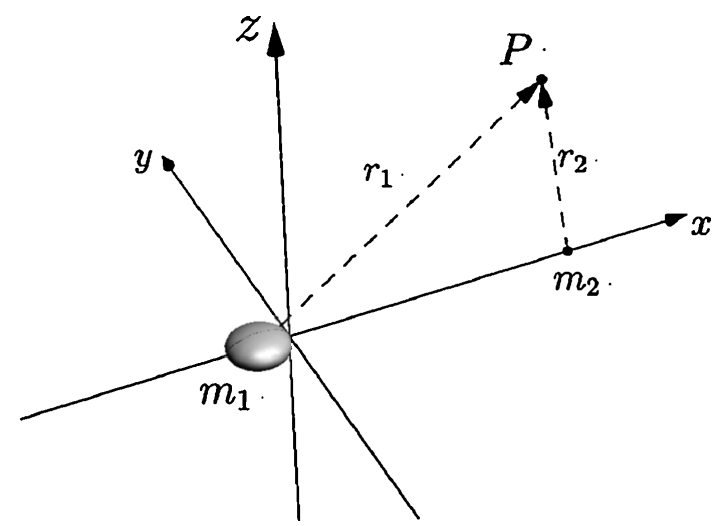

Fig. 1. Illustration of the circular restricted three-body problem with oblateness. The origin of the synodic coordinate is in the barycentre. The primary $m_{1}$ is the rotational ellipsoid whose coordinate is $\left(-\mu_{2}, 0,0\right)$, while $m_{2}$ is the point mass located at $\left(\mu_{1}, 0,0\right)$.

Obviously the gravitational force from the point mass $m_{2}$ (the first term) and the main part of $m_{1}$ always point towards the $X Y$ plane, thus only the component generated by the $J_{2}$ term of $m_{1}$ may balance the acceleration towards the $X Y$ plane. For any out-of-plane point $z \neq 0$, Eq. (4) can be possibly satisfied only if the latter term is negative for some $r_{1}$, i.e.,

$f\left(r_{1}, z\right)=1+\frac{3 A}{2 r_{1}^{2}}\left(3-\frac{5 z^{2}}{r_{1}^{2}}\right)<0$.

Actually, $z^{2}$ can take any value from 0 to $r_{1}^{2}$, and thus the minimum value of $f\left(r_{1}, z\right)$ for arbitrary fixed $r_{1}$ can be reached at $z= \pm r_{1}$, i.e.,

$f_{\min }\left(r_{1}, z\right)=\min _{0 \leq z^{2} \leq r_{1}^{2}} f\left(r_{1}, z\right)=1-\frac{3 A}{r_{1}^{2}}$.

It is necessary to have $f_{\min }\left(r_{1}, z\right)<0$ to make the inequity Eq. (5) true, which gives the estimation of $r_{1}$, that is

$1-\frac{3 A}{r_{1}^{2}}<0 \Longrightarrow r_{1}<\sqrt{3 A}=\sqrt{\frac{3\left(r_{e}^{2}-r_{p}^{2}\right)}{5}}<r_{e}$.

The inequality $r_{1}<r_{e}$ in Eq. (7) means that any out-of-plane equilibrium points obtained by solving Eq. (4) must be located inside the Brillouin sphere around $m_{1}$.

If the rotational ellipsoid satisfies some usual constrains, we can further show that such artificial equilibrium points are even located inside the rotational ellipsoid. Suppose a rotational ellipsoid meets the following conditions:

$r_{p}<r_{e}<\sqrt{\frac{8}{3}} r_{p}$

We first introduce a function $g\left(r_{1}, z\right)$ as follows:

$$
\begin{aligned}
g\left(r_{1}, z\right) & =\left[\frac{\left(x+\mu_{1}\right)^{2}}{r_{e}^{2}}+\frac{y^{2}}{r_{e}^{2}}+\frac{z^{2}}{r_{p}^{2}}\right]-1 \\
& =\frac{\left(r_{1}^{2}-z^{2}\right)}{r_{e}^{2}}+\frac{z^{2}}{r_{p}^{2}}-1,
\end{aligned}
$$

where $\left(x+\mu_{1}\right)^{2} / r_{e}^{2}+y^{2} / r_{e}^{2}+z^{2} / r_{p}^{2}=1$ indicates the surface of the ellipsoid. Then, obviously

$g\left(r_{1}, z\right) \begin{cases}<0, & \text { The point }(x, y, z) \text { is inside the ellipsoid, } \\ >0, & \text { The point }(x, y, z) \text { is outside the ellipsoid. }\end{cases}$

From Eq. (5), we obtain

$\frac{3 A}{2 r_{1}^{2}}\left(3-\frac{5 z^{2}}{r_{1}^{2}}\right)<0 \Longrightarrow \frac{3 r_{1}^{2}}{5}<z^{2} \leq r_{1}^{2}$.

Thus, for any fixed $r_{1}$, the maximum of $g\left(r_{1}, z\right)$ is attained when $z^{2}=r_{1}^{2}$, and thus

$g_{\max }\left(r_{1}, z\right)=\frac{r_{1}^{2}-r_{p}^{2}}{r_{p}^{2}}$.

Since here $g_{\max }$ depends only on $r_{1}$ but not $z$, the estimation given in Eq. (7) shall be taken into account, resulting in

$g_{\max }\left(r_{1}\right)<\frac{3 A-r_{p}^{2}}{r_{p}^{2}}=\frac{3 r_{e}^{2}}{5 r_{p}^{2}}-\frac{8}{5}$.

Because any equilibrium point must satisfy Eqs. (5) and (11), when Eq. (8) is fulfilled, we have for any equilibrium point

$g\left(r_{1}, z\right) \leq g_{\max }\left(r_{1}\right)<0$,

that is, the equilibrium points are inside the ellipsoid. Apparently, such rotational ellipsoid satisfying Eq. (8) is ubiquitous. This gives the counterexamples for the existence of out-of-plane equilibrium because the potential function adopted in Douskos \& Markellos (2006) is obviously invalid inside the ellipsoid.

\section{Discussion}

Base on the force balance in the $Z$ direction, we have analytically shown that the out-of-plane equilibrium points found in Douskos \& Markellos (2006) are located inside the Brillouin sphere of the oblate primary or even below the surface of the ellipsoidal primary body. Since the spherical harmonics expansion of the potential function is not valid at this point, we believe that the existence of these out-of-plane equilibrium points is caused by the improper application of the potential function. In fact, using the closed form of potential function, we can also prove that the acceleration produced by an ellipsoidal asteroid will always point towards to the $O-X Y$ plane, such that no out-of-plane balance point can exist. Surely, this conclusion holds for the case that both primaries are oblate.

Acknowledgements. We thank the anonymous referee for very helpful comments. This work has been supported by the National Natural Science Foundation of China (NSFC, Grants No.11473016 \& No.11333002).

\section{References}

Douskos, C. N., \& Markellos, V. V. 2006, A\&A, 446, 357 